\title{
Desafios do rastreio do câncer de mama em pessoas transgêneros
}

\author{
Challenges of breast cancer screening in transgender \\ Desafíos de la detección del cáncer de mama en personas transgénero
}

\author{
Matheus dos Santos Carvalho \\ ORCID: https://orcid.org/0000-0001-5835-191X \\ Centro Universitário Tiradentes, Brasil \\ E-mail: matheus060913@hotmail.com \\ Mariana Tayná Silva dos Santos \\ ORCID: https://orcid.org/0000-0001-8385-8886 \\ Centro Universitário Tiradentes, Brasil \\ E-mail: mariana.taay@outlook.com.br \\ Pâmela Tays de Holanda Silva \\ ORCID: https://orcid.org/0000-0002-1454-4928 \\ Centro Universitário Tiradentes, Brasil \\ E-mail: pamelatays200@hotmail.com \\ Jaqueline Paulino Gomes \\ ORCID: https://orcid.org/0000-0003-0943-5396 \\ Centro Universitário Tiradentes, Brasil \\ E-mail: Jaqueline.jpg@ hotmail.com \\ Polyana Maccoy e Silva \\ ORCID: https://orcid.org/0000-0002-1981-7884 \\ Centro Universitário Tiradentes, Brasil \\ E-mail: polymaccoy@gmail.com \\ Gêlda Cardoso de Albuquerque \\ ORCID: https://orcid.org/0000-0002-2229-1844 \\ Centro Universitário Tiradentes, Brasil \\ E-mail: geldacardoso@ hotmail.com \\ Natália de Carvalho Lefosse Valgueiro \\ ORCID: https://orcid.org/0000-0001-5761-081X \\ Centro Universitário Tiradentes, Brasil \\ E-mail: profnatalialefosse@gmail.com
}

\begin{abstract}
Resumo
Objetivo: Analisar as evidências da literatura sobre os desafios do rastreio de câncer de mama em homens e mulheres transgêneros. Metodologia: Estudo de revisão integrativa, realizado nas bases de dados: Literatura Latino-Americana e do Caribe em Ciências da Saúde (LILACS), Medical Literature Analysisand Retrieval System Online (MEDLINE), Biblioteca Virtual de Saúde (BVS), Scientific Electronic Library Online (SciELO) e na United States National Library of Medicine (PUBMED). Resultados: A amostra foi composta de onze artigos. Identificou-se que desafios do rastreio do câncer de mama em pessoas transgêneros estão associados as variáveis, como: condição financeira baixa, profissionais descapacitados para o atendimento ao público trans, ausência de uma diretriz específica para o rastreio e serviços de saúde com prática de discriminação. Conclusão: Portanto, faz necessário entender a epidemiologia do câncer supracitado e as necessidades específicas de prevenção e tratamento nas populações transgêneros, que também adote políticas públicas para reduzir estigma e discriminação relacionada a diversidade de gênero.
\end{abstract}

Palavras-chave: Pessoas transgênero; Neoplasias da mama; Programas de rastreamento.

\begin{abstract}
Objective: To analyze evidence from the literature on the challenges of breast cancer screening in transgender men and women. Methodology: Integrative review study, carried out in the following databases: Latin American and Caribbean Literature on Health Sciences (LILACS), Medical Literature Analysis and Retrieval System Online (MEDLINE), Virtual Health Library (VHL), Scientific Electronic Library Online (SciELO) and the United States National Library of Medicine (PUBMED). Results: The sample consisted of eleven articles. It was identified that the challenges of breast cancer screening in transgender people are associated with variables such as: low financial condition, disabled professional to serve the transgender public, absence of a specific guideline for screening and health services with discriminatory practices. Conclusion: Therefore, it is necessary to understand the aforementioned cancer epidemiology and the specific needs for prevention and treatment in transgender populations, which also adopt public policies to reduce stigma and discrimination related to gender diversity.
\end{abstract}

Keywords: Transgender people; Breast neoplasms; Screening programs. 


\begin{abstract}
Resumen
Objetivo: Analizar la evidencia de la literatura sobre los desafíos del cribado del cáncer de mama en hombres y mujeres transgénero. Metodología: Estudio de revisión integrativa, realizado en las siguientes bases de datos: Literatura Latinoamericana y del Caribe en Ciencias de la Salud (LILACS), Sistema de Análisis y Recuperación de Literatura Médica en Línea (MEDLINE), Biblioteca Virtual en Salud (BVS), Biblioteca Electrónica Científica en Línea (SciELO) y la Biblioteca Nacional de Medicina de los Estados Unidos (PUBMED). Resultados: La muestra estuvo conformada por once artículos. Se identificó que los desafíos del cribado de cáncer de mama en personas transgénero están asociados a variables como: baja condición económica, profesionales incapaces de atender al público trans, ausencia de una guía específica para el cribado y servicios de salud con prácticas discriminatorias. Conclusión: Por tanto, es necesario comprender la epidemiología del cáncer antes mencionada y las necesidades específicas de prevención y tratamiento en poblaciones transgénero, que además adoptan políticas públicas para reducir el estigma y la discriminación relacionados con la diversidad de género.
\end{abstract}

Palabras clave: Personas transgénero; Neoplasias mamarias; Programas de cribado.

\title{
1. Introdução
}

Um estudo populacional realizado por Lam e Abramovich (2019) indica que cerca de 0,5\% dos adultos se identificam como transgêneros, que corresponde a 25 milhões de pessoas em todo mundo. De acordo Jesus (2012) define transgênero como um indivíduo que se identifica com um gênero diferente daquele que corresponde ao seu sexo designado no momento do nascimento. Desse modo, esses indivíduos estão sob risco de desenvolver câncer de mama pelos fatores de risco presentes em seus contextos. Com isso, necessitam de cuidados indicados como exames preventivos ou tratamentos (Joint, et al., 2018).

O Instituto Nacional do Câncer define o câncer de mama como uma doença que gera células anormais que se multiplicam formando um tumor. O mesmo não tem uma etiologia única, estando relacionado a diversos fatores que podem aumentar o risco para o desenvolvimento do câncer de mama (INCA, 2020). Os sintomas são edema cutâneo, retração cutânea, dor, inversão do mamilo, hiperemia, descamação ou ulceração do mamilo, secreção papilar, entre outros (Santos \& Chubaci, 2011).

Conforme Silva \& Riul (2011), os fatores de risco aumentam a incidência do câncer de mama como idade, fatores endócrinos, história reprodutiva, fatores comportamentais e fatores hereditários. No entanto, existem fatores de prevenção como praticar atividade física, alimentação saudável, evitar o consumo de bebidas alcoólicas, manter o peso corporal adequado, entre outras estratégias de prevenção (INCA, 2020).

No Brasil, as diretrizes recomendam o início do rastreamento com a mamografia em mulheres sem sinais e sintomas de câncer de mama, na faixa etária de 50 a 69 anos, a mamografia deve ser realizada a cada dois anos. Além disso, o autoexame é uma forma de proporcionar o autoconhecimento e com isso a possibilidade de detecção de lesões palpáveis, ele pode ser feito pelo indivíduo e também pelos profissionais de saúde (Silva, et al., 2017). No entanto, como faltam diretrizes específicas para pacientes Transexuais, Meggetto e colaboradores (2019) recomendam que o rastreio em homens e mulheres trans deve ser feito de acordo com as mesmas diretrizes de mulheres cisgênero.

Entretanto, estudos demonstram que a população trans é muito mais propensa a não estar em dia com os exames preventivos quando comparado a mulheres cisgênero (Joint, et al., 2018; Meggetto, et al., 2019). Estas sofrem diversas dificuldades para ter acesso à saúde de qualidade, porque existe preconceito, estigma e abuso por parte dos profissionais nos serviços de saúde. Sendo assim, esses indivíduos não procuram esses serviços por sofrerem discriminação (Rocon, et., 2016).

A relevância deste estudo está no fato de possibilitar a reflexão sobre a alta mortalidade associada ao câncer de mama e a necessidade de conseguir ampliar o rastreio na população transexual, a qual possui importante prevalência de fatores de risco para essa patologia e baixa taxa de adesão aos exames preventivos diante dos impasses no serviços de saúde. Sendo assim, esse estudo tem como objetivo em analisar as evidências da literatura sobre os desafios do rastreio de câncer de mama em homens e mulheres transgêneros. 


\section{Metodologia}

Trata-se de um estudo descritivo do tipo revisão integrativa. Para a construção desta revisão integrativa, utilizaram-se seis etapas pré-definidas: elaboração da pergunta norteadora; busca na literatura; coleta de dados; análise crítica dos estudos incluídos; discussão dos resultados e apresentação da revisão integrativa (Mendes, et al., 2008).

A primeira fase, referente à pergunta condutora, foi formulada com a estratégia PICO, que significa P para paciente, I para intervenção, C para comparação e O para outcomes (desfecho), teve o seguinte questionamento: Quais os desafios para o rastreamento do câncer de mama em homens e mulheres transgêneros? Posteriormente, seguiu-se para a segunda fase que consistiu na seleção dos estudos (Santos, et al., 2007).

A seleção foi realizada durante os meses de agosto a setembro de 2020 nas bases de dados: Literatura Latino-Americana e do Caribe em Ciências da Saúde (LILACS), Medical Literature Analysisand Retrieval System Online (MEDLINE), Biblioteca Virtual de Saúde (BVS), Scientific Electronic Library Online (SciELO) e na United States National Library of Medicine (PUBMED). Em cada uma dessas bases, foi realizada a busca aos pares utilizando o descritor "Transgender Persons" integrado aos descritores utilizando operador booleano AND "Mass Screening" e "Breast Neoplasm"; e seus análogos em português e em espanhol, todos padronizados pelo Medical Subject Heading (MESH) e Descritores em Ciências da Saúde (DeCS).

A seleção dos artigos ocorreu a partir dos critérios de inclusão: artigo original, escrito nos idiomas Inglês, Português e Espanhol, completos, disponíveis e definiu-se o espaço temporal de 5 anos de publicação. Como critérios de exclusão foram desconsiderados as teses, dissertações, monografias, editoriais, revisões integrativas, sistemáticas e conceituais, bem como a repetição de publicação de estudos em mais de uma base de dados e os artigos que não responderam à questão condutora do estudo.

Em seguida, os estudos foram avaliados quanto ao nível de evidência, baseado na categorização, em conformidade com a abordagem metodológica da Agency for Healthcare Research and Quality (AHRQ), a saber: Nível I - Revisões sistemáticas ou metanálise de ensaios clínicos relevantes; Nível II - Ensaio clínico randomizado controlado bem delineado; Nível III - Ensaios clínicos bem delineados sem randomização; Nível IV - Estudos de coorte e de caso-controle bem delineados; Nível V - Revisão sistemática de estudos descritivos e qualitativos; Nível VI - Evidências derivadas de um único estudo descritivo ou qualitativo; Nível VII - Opinião de autoridades ou relatório de comitês de especialista (Galvão, 2016).

A estratégia de busca resultou em 43387 artigos, distribuídos nas bases de dados determinadas. Os artigos duplicados foram retirados e contabilizados em apenas uma base de dados, sendo considerado na base que apresentava maior facilidade no acesso, sendo exposto na Tabela 1. 
Tabela 1 - Artigos encontrados nas referidas bases de dados, biblioteca virtual com seus cruzamentos, Recife (PE), Brasil, 2020.

\begin{tabular}{|c|c|c|c|c|c|c|}
\hline & $\begin{array}{c}\text { Cruzamentos } \\
\text { busca }\end{array}$ & MEDLINE & SCIELO & BVS & LILACS & PUBMED \\
\hline $1^{\circ}$ & $\begin{array}{l}\text { "Transgender } \\
\text { Persons" AN } \\
\text { D "Breast } \\
\text { Neoplasm" }\end{array}$ & 52 & 00 & 53 & 01 & 52 \\
\hline $2^{\circ}$ & $\begin{array}{l}\text { "Transgender } \\
\text { Persons" } \\
\text { AND "Mass } \\
\text { screening" }\end{array}$ & 121 & 00 & 117 & 00 & 114 \\
\hline $3^{\circ}$ & $\begin{array}{c}\text { "Breast } \\
\text { Neoplasm" } \\
\text { AND "Mass } \\
\text { Screening" }\end{array}$ & 14793 & 02 & 15398 & 122 & 12553 \\
\hline $4^{\circ}$ & $\begin{array}{l}\text { "Transgender } \\
\text { Persons " AN } \\
\text { D "Breast } \\
\text { Neoplasm" } \\
\text { AND "Mass } \\
\text { Screening" }\end{array}$ & 5 & 00 & 04 & 00 & 00 \\
\hline TOTAL & 43387 & 14971 & 02 & 15572 & 123 & 12719 \\
\hline
\end{tabular}

Fonte: Autores (2020).

Os títulos e os resumos destes artigos foram lidos, sendo incluídos, apenas, aqueles que abrangiam a temática do estudo e excluindo-se os que não correspondiam aos critérios de elegibilidade previamente estabelecidos, tendo havido a redução da amostra para 62 artigos. Destes, foi realizada a avaliação dos artigos, lidos todos na íntegra e os que não respondiam a questão norteadora foram retirados, restando apenas $\mathbf{1 1}$ artigos como amostra final. 
Figura 1 - Fluxograma do processo de seleção dos artigos para revisão integrativa, 2020.

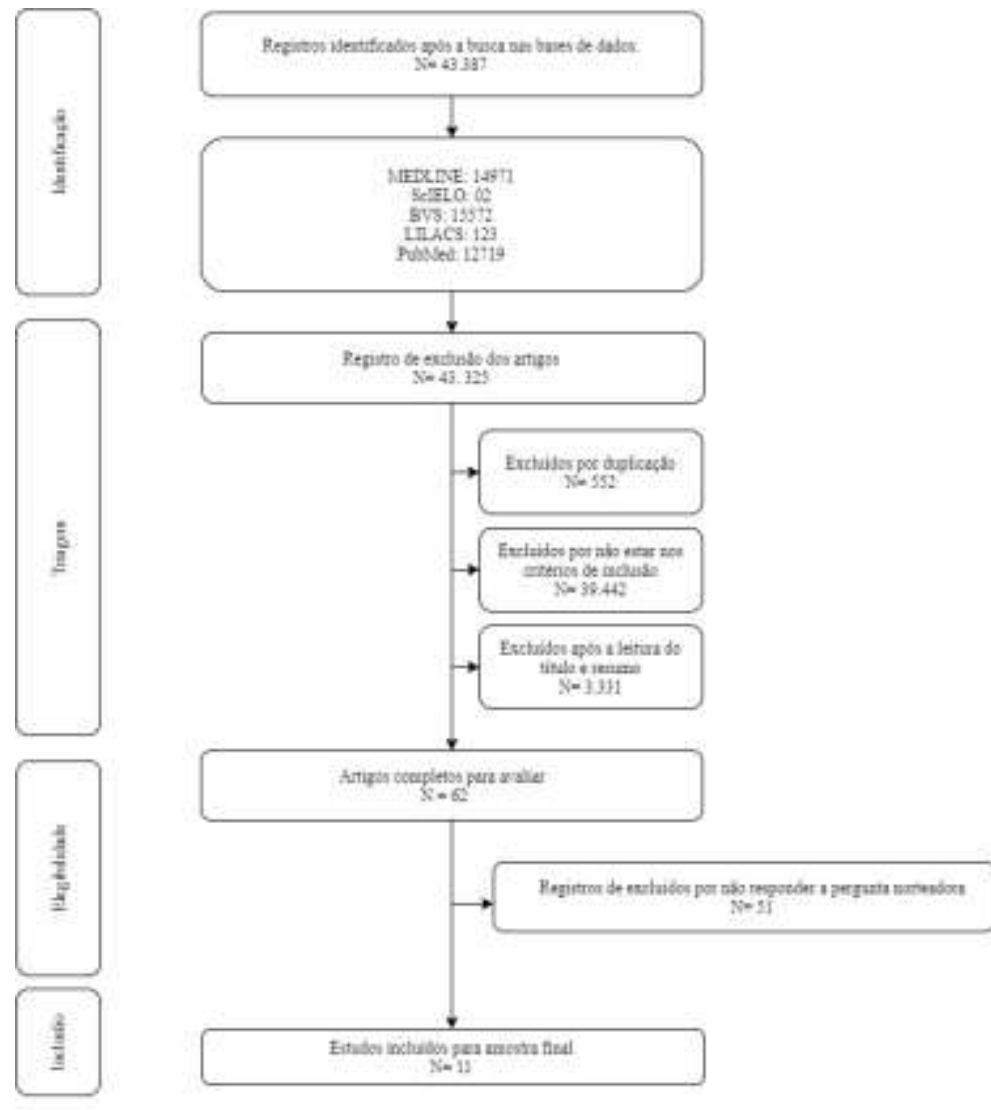

Fonte: Autores (2020).

O instrumento utilizado para sintetizar os resultados foi o proposto Ursi (2005). O preenchimento do instrumento foi realizado por dois revisores de forma independente, para extração dos principais aspectos abordados. Na interpretação dos resultados, seguiu-se à leitura comparativa entre os artigos, verificando-se suas similaridades, procedendo-se ao agrupamento e análise de conteúdo (Bradin, 2010).

\section{Resultados}

Os resultados apresentados no Quadro 1, pautaram-se em 11 publicações, das quais cinco foram obtidas na MEDLINE, uma na LILACS, uma na BVS e quatro na PUBMED. Os idiomas encontrados nos estudos foram inglês e espanhol. Os países onde foram realizados os estudos foram Argentina, Canadá, Estados Unidos, Sérvia, Reino Unido e Singapura. O período de publicação dos mesmos ocorreu entre os anos de 2015 a maio de 2020. Dessa forma, no Quadro 1, as publicações foram organizadas em: autores e ano, título, objetivo e resultados. 
Quadro 1 - Descrição dos artigos que abordam os desafios para o rastreio do câncer de mama em homens e mulheres transgêneros.

\begin{tabular}{|c|c|c|c|c|}
\hline Autor / Ano & Título & $\begin{array}{c}\text { Nível de evidência / } \\
\text { País }\end{array}$ & Objetivo & Resultados encontrados \\
\hline $\begin{array}{l}\text { Lienhoop et al, } \\
2020 .\end{array}$ & $\begin{array}{l}\text { Breast cancer in } \\
\text { transgender women: } \\
\text { A case report. }\end{array}$ & $\begin{array}{l}\text { Nível VI / Estudos } \\
\text { Unidos }\end{array}$ & $\begin{array}{l}\text { Relatar um caso de câncer de mama em } \\
\text { uma mulher trans em terapia hormonal. }\end{array}$ & $\begin{array}{l}\text { Retrata que mulheres transgêneros têm mais risco } \\
\text { para o câncer de mama em relação as mulheres } \\
\text { cisgênero, porém os profissionais não realizam o } \\
\text { rastreio do câncer de mama por falta de diretrizes } \\
\text { específicas e despreparo profissional. }\end{array}$ \\
\hline $\begin{array}{l}\text { Bazzi et al, } \\
2015 .\end{array}$ & $\begin{array}{l}\text { Adherence to } \\
\text { Mammography } \\
\text { Screening } \\
\text { Guidelines Among } \\
\text { Transgender Persons } \\
\text { and Sexual Minority } \\
\text { Women. }\end{array}$ & $\begin{array}{l}\text { Nível VI / Estados } \\
\text { Unidos }\end{array}$ & $\begin{array}{l}\text { Analisar a adesão da população } \\
\text { transgênero e mulheres em minorias } \\
\text { sexuais na diretriz da mamografia. }\end{array}$ & $\begin{array}{l}\text { Expõe que homens e mulheres trans aderem em } \\
\text { índices baixos às diretrizes da mamografia, } \\
\text { porque não se sentem acolhidos nos serviços de } \\
\text { saúde. }\end{array}$ \\
\hline $\begin{array}{l}\text { Kiran et al, } \\
2019 .\end{array}$ & $\begin{array}{lr}\text { Cancer } & \text { screening } \\
\text { rates } & \text { among } \\
\text { transgender adults. }\end{array}$ & Nível IV / Canadá & $\begin{array}{l}\text { Comparar as taxas de rastreamento do } \\
\text { câncer cervical, de mama e colorretal } \\
\text { entre } \\
\text { pacientes transgêneros e cisgêneros (ou } \\
\text { seja, não transgêneros). }\end{array}$ & $\begin{array}{l}\text { Esse estudo aborda que a população trans tem uma } \\
\text { baixa adesão comparada com a população } \\
\text { cisgênero. Além disso, relata que as condições } \\
\text { financeiras em nível baixo são um contexto para o } \\
\text { baixo rastreamento do câncer de mama nos } \\
\text { indivíduos trans. }\end{array}$ \\
\hline Pivo et al, 2016. & $\begin{array}{lr}\text { Breast cancer } & \text { risk } \\
\text { assessment } & \text { and } \\
\text { screening } & \text { in } \\
\text { transgender patients. }\end{array}$ & $\begin{array}{l}\text { Nível VI / Estados } \\
\text { Unidos }\end{array}$ & $\begin{array}{l}\text { Avaliar a triagem de risco de câncer de } \\
\text { mama em pacientes transsexuais. }\end{array}$ & $\begin{array}{l}\text { Devido a ausência de uma diretriz formal sobre o } \\
\text { rastreio do câncer de mama, causa uma } \\
\text { negligência dos serviços para com esse público e } \\
\text { sua adesão. }\end{array}$ \\
\hline $\begin{array}{l}\text { Chotai et al, } \\
2018 .\end{array}$ & $\begin{array}{l}\text { Breast cancer in a } \\
\text { female to male } \\
\text { transgender patient } \\
20 \text { years post } \\
\text { mastectomy: Issues } \\
\text { to consider. }\end{array}$ & Nível VI / Singapura & $\begin{array}{l}\text { Relatar o caso de um homem trans que } \\
\text { teve câncer de mama após a } \\
\text { mastectomia. }\end{array}$ & $\begin{array}{l}\text { Demonstra-se que a dificuldade no rastreio do } \\
\text { câncer de mama na população transgênero é por } \\
\text { conta do estigma e preconceito nos serviços de } \\
\text { saúde. }\end{array}$ \\
\hline $\begin{array}{l}\text { Castro et al, } \\
2019 .\end{array}$ & $\begin{array}{l}\text { Screening mamario } \\
\text { en pacientes } \\
\text { transgénero bajo } \\
\text { tratamiento } \\
\text { hormonal cruzado } \\
\text { (thc). Situación } \\
\text { actual } \\
\text { controversias }\end{array}$ & Nível VI / Argentina & $\begin{array}{l}\text { Estudar a situação atual em relação a } \\
\text { triagem mamária realizada em pacientes } \\
\text { trans por seus clínicos gerais em } \\
\text { hospital da cidade de Buenos Aires. }\end{array}$ & $\begin{array}{l}\text { Apresenta que a falta de uma diretriz para o } \\
\text { rastreio do câncer de mama em pessoas } \\
\text { transgênero, causa uma negligência pelo fato da } \\
\text { população trans está sob maior risco, porém } \\
\text { menos assistida nos serviços de saúde. }\end{array}$ \\
\hline $\begin{array}{l}\text { Macdonald, } \\
2019 .\end{array}$ & $\begin{array}{l}\text { Managing breast } \\
\text { cancer risk in } \\
\text { transgender men and } \\
\text { women: Emerging } \\
\text { safety data and a } \\
\text { need for more } \\
\text { research. }\end{array}$ & $\begin{array}{l}\text { Nível VII / Estados } \\
\text { Unidos }\end{array}$ & $\begin{array}{l}\text { Avaliar o gerenciamento do risco do } \\
\text { câncer de mama em homens e mulheres } \\
\text { transgêneros. }\end{array}$ & $\begin{array}{l}\text { Nesse estudo os desafios perpetuam sobre os } \\
\text { medos que os pacientes têm em ser maltratados } \\
\text { nos serviços de saúde, a falta de capacitação dos } \\
\text { profissionais para o atendimento ao público trans } \\
\text { e em indivíduos com condição financeira baixa. }\end{array}$ \\
\hline $\begin{array}{l}\text { Narayan et al, } \\
2017 .\end{array}$ & $\begin{array}{l}\text { Breast cancer } \\
\text { screening in } \\
\text { transgender patients: } \\
\text { findings from the } \\
\text { 2014 BRFSS survey. }\end{array}$ & $\begin{array}{llll}\text { Nível } & \text { IV } / \text { Reino } \\
\text { Unido } & & & \\
\end{array}$ & $\begin{array}{l}\text { Determinar até que ponto as pacientes } \\
\text { trans são submetidas a exames de } \\
\text { mamografia usando dados } \\
\text { de pesquisa representativos a nível } \\
\text { nacional. }\end{array}$ & $\begin{array}{l}\text { Apresenta que há uma baixa adesão no } \\
\text { rastreamento por conta da discriminação por parte } \\
\text { dos profissionais de saúde, mas também em } \\
\text { indivíduos trans que tem uma condição financeira } \\
\text { baixa. }\end{array}$ \\
\hline $\begin{array}{l}\text { Nisly et al, } \\
2018 \text {. }\end{array}$ & $\begin{array}{l}\text { Unique Primary } \\
\text { Care Needs of } \\
\text { Transgender and } \\
\text { Gender Non-Binary } \\
\text { People. }\end{array}$ & $\begin{array}{l}\text { Nível VI / Estados } \\
\text { Unidos }\end{array}$ & $\begin{array}{l}\text { Descrever as necessidades da população } \\
\text { transgênero e não binária nos serviços } \\
\text { de atenção a saúde primária. }\end{array}$ & $\begin{array}{l}\text { Aborda que as pessoas trans não buscam os } \\
\text { serviços para o rastreio do câncer de mama, } \\
\text { devido a humilhação, exposição e ofensa por } \\
\text { conta da identidade de gênero. }\end{array}$ \\
\hline $\begin{array}{l}\text { Nikolić et al, } \\
2018 \text {. }\end{array}$ & $\begin{array}{l}\text { Breast cancer and its } \\
\text { impact in male } \\
\text { transsexuals. }\end{array}$ & Nível VI / Sérvia & $\begin{array}{l}\text { Descrever os impactos do câncer de } \\
\text { mama em transexuais masculinos. }\end{array}$ & $\begin{array}{l}\text { Demonstra como fator de dificuldade para o } \\
\text { rastreio é a violência verbal por profissionais nos } \\
\text { serviços de saúde. }\end{array}$ \\
\hline $\begin{array}{l}\text { Zhirui et al, } \\
2017 \text {. }\end{array}$ & $\begin{array}{l}\text { Cancer prevention } \\
\text { and screening in a } \\
\text { BRCA2 positive } \\
\text { male to female } \\
\text { transgender patient. }\end{array}$ & Nível VII / Canadá & $\begin{array}{l}\text { Descrever contextos de prevenção e } \\
\text { rastreio do câncer de mama na } \\
\text { população transgênero. Além disso, } \\
\text { relata um estudo sobre o câncer de } \\
\text { mama em uma mulher trans. }\end{array}$ & $\begin{array}{l}\text { A discriminação por profissionais, falta de dados } \\
\text { e diretrizes específicas são apontados como as } \\
\text { principais dificuldades para o rastreio do câncer } \\
\text { de mama na população transgênero. }\end{array}$ \\
\hline
\end{tabular}

Fonte: Autores (2020).

Nos 11 artigos selecionados e lidos na íntegra entre os seus achados deixa claro que as questões dos desafios do rastreio do câncer de mama em homens e mulheres transgêneros estão associados as variáveis, tais como: condição financeira baixa, 
profissionais descapacitados para o atendimento ao público trans, ausência de uma diretriz formal e serviços de saúde com prática de discriminação.

$\mathrm{Na}$ categoria I evidencia-se que os desafios encontrados para o rastreio nessa população foram os profissionais sem capacitação para atender as demandas e necessidades específicas desse público. Somando a isso, o quão essa descapacitação influência nos serviços, pois resulta em discriminação e imposição dos valores heterocisnormativo pelos profissionais para com as identidades trans que buscam os serviços.

Na categoria II notou-se que um outro desafio está associado à questão financeira dessa população, pelo fato de que alguns países possuem os serviços de saúde pagos e alguns indivíduos trans marginalizados não conseguem ter acesso. Desse modo, buscam os serviços de saúde quando o câncer já se encontra em estado grave.

Na categoria III demonstra-se que a ausência de diretriz específica para o rastreio nesse público torna-se uma desafio, pois é frequentemente usada a diretriz da população cisgênera para o rastreio no público trans, sendo que as realidades de vivências trans não são conforme o das pessoas cis. Consequentemente, não há uma padronização para que todos os profissionais sigam no rastreio nesse público supracitado, tornando-se algo não sistematizado que gera negligência.

\section{Discussão}

\section{Categoria I - Discriminação nos serviços de saúde e ausência de capacitação dos profissionais}

No estudo de Bazzi e colaboradores (2015) aborda que a população mencionada no estudo não se sente acolhida nos serviços de saúde, sendo esse contexto uma dificuldade para o rastreio, porque essa percepção resulta no afastamento desse público nos serviços supracitados. Chotai e colaboradores (2018) corrobora abordando que o preconceito é um dos fatores para que homens e mulheres transgêneros não se sintam acolhidos nos serviços de saúde.

Nisly e colaboradores (2018) demonstram que essa discriminação acontece com humilhação, julgamento moral e preconceito pela identidade gênero das pessoas trans. Somando a isso, o estudo de Nicolić\& colaboradores (2018) relata que essa discriminação é resultante do estigma social que é trazido para o ambiente de saúde, tendo como resultado a não possibilidade de garantias de direito à saúde.

Macdonald (2019) descreve que essas discriminações geram medo, sofrimento psíquico e negação ao acesso à saúde, fazendo com que esse público dificilmente se dirija às instituições de saúde, mesmo em casos graves. Consequentemente, essa população busca os serviços clandestinos como uma outra alternativa (Pereira \& Chazan,2019).

O preconceito afasta esse público dos serviços de saúde, automaticamente não buscando cuidados de prevenção e promoção da saúde, tornando-os vulneráveis a algumas doenças (Rocon, et al., 2016). De acordo com Lienhoop e colaboradores (2020), as pessoas transgêneras estão sob mais riscos do câncer de mama, mas com pouca aderência da mamografia, devido o "afastamento" causado pelo preconceito nos serviços mencionados neste texto.

Além disso, o estudo de Silva e colaboradores (2020) expõe que os profissionais de saúde no processo de formação não são ensinados sobre gênero e sexualidade, e quando são ensinados é no contexto heterocisnormativo que não engloba toda diversidade existente de identidades de gênero e orientações sexuais. Com isso, o estudo de Souza e colaboradores (2016) debate que o fato de não conhecer essa diversidade enquanto acadêmico ou profissional é uma grande problemática, visto que esses profissionais na assistência de saúde impõem valores heterocisnormativo para as identidades trans, que promove discriminação estrutural.

Segundo Lienhoop e colaboradores (2020) quando os profissionais são capacitados para o atendimento ao público trans, os serviços são mais acolhedores e as discriminações são amenizadas. Dessa maneira, Santana e colaboradores (2020) relatam que além de uma capacitação, a temática de saúde transgênera deve ser abordada no processo de formação acadêmica, pois 
auxilia enquanto futuros profissionais a abordar as necessidades e demandas específicas de saúde para esse público nos serviços supracitados.

A capacitação auxilia os servidores a compreender melhor as demandas das pessoas trans pelo fato de possibilitar a compreensão da diversidade em relação a subjetividade e singularidade dos sujeitos. Sendo necessário estar disposto a ouvir e buscar o conhecimento para entender as diferenças e acima de tudo respeitá-las (Abade, et al., 2020). Somando a isto, o estudo de Santana e colaboradores (2020) expõe que além da capacitação é necessário a desconstrução dos profissionais em relação aos estereótipos de gênero engendrados pela heterocisnormatividade e legitimadas por vários discursos inclusive na saúde que consideram as pessoas trans erradas por serem "divergentes" do padrão cisgênero, que inclusive é aceito nas instituições.

\section{Categoria II - Marginalização e pobreza}

De acordo com a Associação de Travestis e Transexuais - ANTRA, cerca de 90\% da classe sobrevivem de trabalhos informais e marginalizados (Otoni, 2014). Esse dado também é equivalente quando trata-se da realidade de países desenvolvidos, pois há uma imposição social que impede que pessoas transgêneras sejam inseridas no ambiente de trabalho e as impossibilitam em frequentar cursos profissionalizantes decorrido da evasão escolar que expulsa esse público por não acolhimento nessas instituições de ensino (Pereira \& Gomes, 2017).

Nesse contexto, algumas pessoas trans buscam a prostituição como um meio de sustento e sobrevivência (Rocon, et al., 2016). Diante disso, é necessário problematizar a situação do público supracitado em países que possuem os serviços de saúde sem gratuidade, o que difere do Brasil que possui o Sistema Único de Saúde (SUS), que garante gratuidade de atendimento a todos os cidadãos (Brasil, 1988). Os indivíduos trans na prostituição não ganham um lucro ideal para manter suas necessidades básicas, tendo incapacidade em frequentar os serviços de saúde em países sem gratuidade, pois a marginalização não possibilita colocar a saúde no contexto de necessidade básica (Pereira \& Gomes, 2017).

Consequentemente, o estudo de Macdonald (2019) expõe que a questão financeira e a marginalização tornam-se um desafio para o rastreio do câncer de mama, pois como não é frequente usufruir dos serviços mencionados por não possuir condição para pagar pelos mesmos, esse público só busca as instituições de saúde quando o estado da doença se encontra de forma avançada e grave. Não sendo frequente a busca por consultas e exames preventivos por não conseguir arcar com as despesas destes. A vista disso, essa população está em vulnerabilidade da própria sobrevivência, pois colocam prioridades de acordo com a questão financeira e a saúde fica como algo subalterno (Nayaran, et al., 2017).

\section{Categoria III - Câncer de mama e ausência de diretriz específica para transexuais}

Segundo Migowski e Corrêa (2021), o câncer de mama é mais frequente por volta de 50 e 60 anos de idade. Dessaforma, um grande quantitativo da população transgênera ainda não se encontra na faixa etária de risco, pois o público apresenta uma expectativa de vida menor quando comparado com a população geral por contexto econômico, político, social e de saúde (Jesus, 2012). Consequentemente, isso pode ser a justificativa para poucos estudos e casos de câncer de mama descritos nesses indivíduos, visto que a expectativa de vida é de 35 anos em transexuais (Otoni, 2014).

Com os baixos índices de estudos, o estudo de Zhirui e colaboradores (2017) demonstrou que os homens trans têm cerca de $2 \%$ de risco para o câncer de mama e as mulheres trans têm cerca de $2 \%$ a $3 \%$ de risco para o câncer de mama. Sendo necessário ressaltar que esses valores são baixos quando comparado com os índices das pessoas cisgêneras (Santos \& Chubaci, 2011).

É necessário discutir os fatores de risco para além da idade, fatores endócrinos, fatores comportamentais e fatores genéticos (Adamia,et al., 2008). Na população transgênera também existe a condição da hormonioterapia que há uma controvérsia se é um fator de proteção ou de risco para o câncer mencionado. Segundo Kiran \& colaboradores (2019), o hormônio 
feminino usado por mulheres transgêneros aumenta o risco do câncer exposto, seja ele com ou sem bloqueadores de androgênios. Castro e colaboradores (2019) corroboram e acrescentam que os homens trans que realizaram a mastectomia têm índices baixos para o câncer supracitado. Entretanto, descreve que o hormônio masculino aumenta o risco para o câncer mencionado pelo fato de que uma parte do hormônio é convertido em hormônios femininos e alguns tumores podem ter receptores de androgênio.

É notório os baixos índices de pesquisas científicas na área da saúde que abordem especificidades da população trans de forma integral (Oliveira \& Romanini, 2020). Logo, os poucos estudos existentes sobre o câncer de mama na população supracitada não são capazes de nortear uma diretriz específica para esse público. Dessa maneira, Lienhoop e colaboradores (2020) abordam que a ausência de uma diretriz específica ocasiona uma negligência, pois os profissionais realizam o rastreio de forma não sistematizada, observou que alguns serviços analisado em seu estudo incluía esse público, enquanto outros serviços não os incluía no rastreamento precoce.

O estudo de Castro e colaboradores (2019) e Pivô e colaboradores (2016) expõem que a ausência de diretriz específica é um desafio para o rastreio do câncer de mama na população transgênera. As realidades vividas por pessoas trans não são equivalentes às das pessoas cisgêneras, e essas realidades distintas não podem ser igualadas em única diretriz. Zhirui e colaboradores (2018) relatam que uma diretriz específica para o público trans possibilita os profissionais de saúde no melhor entendimento das vivências desses indivíduos e como podem integrar o rastreio, diagnóstico e tratamento eficaz sem associar essa população ao contexto cisgênero que é totalmente diferente em vivências.

Portanto, como ainda faltam diretrizes específicas para pacientes transexuais, a American College of Radiology (ACR) recomenda que o rastreio em homens e mulheres transgêneros deve ser realizado da mesma forma que as pessoas cisgêneras. Sendo assim, amamografia de rastreamento, que é o exame de rotina em pessoas sem sinais e sintomas de câncer de mama, é recomendada na faixa etária de 50 a 69 anos, a cada dois anos. Fora dessa faixa etária e dessa periodicidade, os riscos aumentam e existe maior incerteza sobre benefícios. No entanto, no caso de homens trans que fizeram a mastectomia não precisam realizar a mamografia, sendo necessário apenas exame clínico das mamas (Migowski \&Corrêa, 2021).

\section{Considerações Finais}

Com este estudo foi possível evidenciar na literatura científica nacional e internacional, os desafios para o rastreio do câncer de mama em homens e mulheres transgêneros, os quais foram extraídos das investigações dos artigos produzidos nos últimos cinco anos. A metodologia empregada propiciou as evidências desses elementos e o aprofundamento teórico sobre as questões referentes à temática. Desta forma, considera-se que este método se mostrou eficaz para a efetivação do objetivo.

Devido ao contexto de discriminação, no qual homens e mulheres transgêneros estão inseridos, percebe-se que os serviços de saúde também perpetuam esse contexto dentro da assistência. Os resultados apresentados ao longo da revisão mostraram que os desafios estão na falta da capacitação dos profissionais, consequentemente não sabem lidar com as especificidades dessa população. Além disso, a ausência de política pública de saúde para o rastreio, ocasionam negligência no fator de prevenção, detecção e tratamento.

Portanto, se faz necessário entender a epidemiologia do câncer de mama e as necessidades específicas de prevenção e tratamento nas populações transgêneras e que se adote políticas públicas para reduzir estigma e discriminação relacionada a diversidade de gênero. Dessa forma, espera-se que este conhecimento sirva de base para solidificar ações para enfrentamento desses desafios e maior acolhimento da população transgênero na atenção básica. Diante disso, existe a necessidade para próximos estudos que abordem um quantitativo maior dessa população relacionada a temática, que estudos com essa população associada ao câncer de mama também sejam feitos no Brasil e por fim, uma construção de uma política pública de saúde que amenize esses desafios. 


\section{Referências}

Abade, E. A. F., Chaves, S. C. L., \& Silva, G. C. O. (2020). Saúde da população LGBT: uma análise dos agentes, dos objetos de interesse e das disputas de um espaço de produção científica emergente. Revista de Saúde Coletiva, 30 (4), 1-31.

Bradin, L. (2010). Análise de conteúdo. (4 ed.). Ed Lisboa.

Brazzi, A. R., Whorms, D. S., King, D. S., \& Potter, J. (2015). Adherence to Mammography Screening Guidelines Among Transgender Persons and Sexual Minority Women. American Journal Of Public Health, 55 (11), 2356-2358.

Castro, A. D., \&Nagelberg, A. (2019). Triagem mamario en pacientes transgénero bajo tratamiento hormonal cruzado (thc): Situação atual e controvérsias. Revista Argentina de Mastología, 38 (137), 116-132.

Chotai, N., Tang, S., Lim, H., \& Lu, S. (2019). Câncer de mama em uma paciente transgênero do sexo feminino para masculino 20 anos após a mastectomia: questões a serem consideradas. The Breast Journal, 25 (6), 1066-1070.

Jesus, J. G. (2012). Orientações sobre identidade de gênero: conceitos e termos. Revista e Ampliada, 2 (1), 1-42.

Joint, R., Chen, Z., \& Cameron, S. (2018). Breast and reproductive cancers in the transgender population: a systematic review. International Journal of Obstetrics \& Gynaecology, 125 (12), 1505-1512.

Kiran, T., Davie, S., Singh, D., Hranilovic, S., Pinto, A. D., Abramovich, A., \&Lofters, A. (2019). Cancer screening rates among transgender adults. Canadian Family Physician, 65 (7), 30-37.

Lam, J. S. H., \& Abramovich, A. (2019). Transgender-inclusive care. Canadian Medical Association Journal, 191 (3), $79-79$.

Li, J. Z., Ti, H. Y. V., Avram, R., Pinthus, J., Bordeleau, L., \&Hodgson, N. (2018). Cancer prevention and screening in a BRCA2-positive male to female transgender patient. The Breast Journal, 24 (6), 1112-1113.

Lienhoop, T., Smetko, M., \& Green, L. (2020). Breast cancer in transgender women: a case report. Clinical Imaging, 68, 20-23.

Macdonal, H. R. (2019). Managing breast cancer risk in transgender men and women: emerging safety data and a need for more research. The Breast Journal, 25 (6), 1063-1065.

Meggetto, O., et al. (2019). Breast cancer risk and breast screening for trans people: an integration of 3 systematic reviews. Cmaj Open, 7 (3), 598-609.

Mendes, K. D. S., Silveira, R. C. C. P., \& Galvão, C. M. (2008). Revisão integrativa: método de pesquisa para a incorporação de evidências na saúde e na enfermagem. Texto \& Contexto - Enfermagem, 17 (4), 758-764.

Nayaran, A., et al. (2017). Breast cancer screening in transgender patients: findings from the 2014 brfss survey. Breast Cancer Research And Treatment, 166 (3), 875-879.

Nikolić, D., et al. (2018). Breast cancer and its impact in male transsexuals. Breast Cancer Research And Treatment, 171 (3), 565-569.

Nisly, N. L., et al. (2018). Unique Primary Care Needs of Transgender and Gender Non-Binary People. Clinical Obstetrics And Gynecology, 61 (4), 674-686.

Otoni, I. (2014). O preconceito afasta as pessoas transgêneros da escola, reduz oportunidades de trabalho e abre as portas da prostituição. Revista Fórum Digital Semanal, 132, 1-14.

Pereira, F. Q., \& Gomes, J. M. (2017). Pobreza E Gênero: a marginalização de travestis e transexuais pelo direito. Revista Direitos Fundamentais \& Democracia, $22(2), 210$.

Pereira, L. B. C., \& Chazan, A. C. S. (2019). O Acesso das Pessoas Transexuais e Travestis à Atenção Primária à Saúde: uma revisão integrativa. Revista Brasileira de Medicina de Família e Comunidade, 14 (41), 1795-1802.

Pivo, S., et al. (2017). Breast Cancer Risk Assessment and Screening in Transgender Patients. Clinical Breast Cancer, 17 (5), $225-227$.

Rocon, P. C., et al. (2016). Dificuldades vividas por pessoas trans no acesso ao Sistema Único de Saúde. Ciência \& Saúde Coletiva, 21 (8), $2517-2526$.

Santana, A. D. S., et al. (2020). Dificuldades no acesso aos serviços de saúde por lésbicas, gays, bissexuais e transgêneros. Revista de Enfermagem UfpeOnLine, $14,1-11$.

Santos, C. M. C., Pimenta, C. A. M., \& Nobre, M. C. (2007). The PICO strategy for the research question construction and evidence search. Revista LatinoAmericana de Enfermagem, 15 (3), 508-511.

Santos, G. D., \& Chubaci, R. Y. S. (2011). O conhecimento sobre o câncer de mama e a mamografia das mulheres idosas frequentadoras de centros de convivência em São Paulo (SP, Brasil). Ciência \& Saúde Coletiva, 16 (5), 2533-2540.

Silva, G. A., et al. (2017). Early detection of breast cancer in Brazil: data from the national health survey, 2013. Revista de Saúde Pública, 51 (1), 1-9.

Silva, J. M. N., Paulino, D. B., \& Raimondi, G. A. (2020). Gênero e Sexualidade na Graduação em Saúde Coletiva do Brasil. Ciência \& Saúde Coletiva, 25 (6), 2335-2346.

Silva, P. A., \& Riul, S. S. (2011). Câncer de mama: fatores de risco e detecção precoce. Revista Brasileira de Enfermagem, 64 (6), $1016-1021$.

Ursi, E. S. (2005). Prevenção de lesões de pele no perioperatório: revisão integrativa da literatura. Dissertação (Mestrado) - Curso de Enfermagem, c130. 